\title{
Dissociation of Different Conformations of Ubiquitin Ions
}

\author{
Ethan R. Badman, Cherokee S. Hoaglund-Hyzer, and David E. Clemmer \\ Department of Chemistry, Indiana University, Bloomington, Indiana, USA
}

\begin{abstract}
The fragmentation pathways of different conformations of three charge states of ubiquitin ions are examined using ion mobility/collisional activation/time-of-flight techniques. Mass spectra for fragments for different conformers of a single charge state appear to be identical (within the experimental reproducibility). These results are consistent with a mechanism in which different conformers of each charge state rearrange to similar dissociation transition states prior to fragment formation. (J Am Soc Mass Spectrom 2002, 13, 719-723) (C) 2002 American Society for Mass Spectrometry
\end{abstract}

$\mathrm{T}$ he ability to produce macromolecules that contain non-covalent structure in the gas phase with mass spectrometric techniques is of significant interest, especially in terms of understanding behavior in solution. However, an understanding of the relationship between gas phase structure and solution conformation is still at a very primitive stage, in large part because detailed gas phase structural information is limited. In addition to chemical probes of gas phase ion structure [1-14], a number physical methods are being developed to examine gas-phase peptide and protein ion structure [15-27], including collisional activation to examine fragmentation pathways [18-23, 25-27]. A few studies have suggested that ion conformation may influence fragmentation pathways [19, 23, 27, 28]. In this paper, we examine this issue for the fragmentation of a series of compact and elongated conformations of ubiquitin ions. The different conformations of ubiquitin are separated in a drift tube prior to collisional activation in a gas cell. Under all experimental conditions that we have employed, dissociation patterns for different conformations within a given ion charge appear to be identical.

Ubiquitin was the first protein chosen for these studies because of its relatively small size, simple structure without disulfide linkages, and because of the extensive data in the literature from dissociation experiments $[17,27,29-38]$ as well as ion mobility studies [24, 39]. Ubiquitin (bovine) is a small protein of 76 amino acids $\left(\mathrm{H}_{2} \mathrm{~N}\right.$-MQIFVKTLTG KTITLEVEPS DTIENVKAKI QDKEGIPPDQ QRLIFAGKQL EDGRTLSDYN IQKESTLHLV LRLRGG-COOH) with 13 basic sites (7 lysine, 4 arginine, 1 histidine, and the amino terminal group) where protonation can occur.

Published online April 26, 2002

Address reprint requests to Dr. D. E. Clemmer, Department of Chemistry, Indiana University, Bloomington, IN 47405, USA. E-mail: clemmer@ indiana.edu
These present studies are related to interesting issues that arise in statistical dissociation theories such as those developed by Rice Ramsperger Kassel and Marcus (RRKM) theory [40,41]. RRKM theory assumes that energy imparted to the molecule will be partitioned statistically among the accessible internal degrees of freedom. For small molecules with relatively few degrees of freedom, this treatment can provide information about the rates of specific reaction channels. However, in the case of a large system, such as a protein, one might imagine that energy transfer among various types of secondary structure could be slower than dissociation steps. In such cases, different conformations might dissociate along different potential surfaces (or access them at different rates). Our present results suggest that any such distinctions between the initial conformations of ubiquitin are lost prior to the fragmentation process.

\section{Experimental}

Experiments were performed with a quadrupole ion trap/drift tube/linear quadrupole/octopole collision cell/time-of-flight (TOF) instrument with electrospray ionization described previously [42]. Briefly, positive ubiquitin (bovine, Sigma, $>95 \%$ ) ions were formed by electrospray ionization of a $3 \times 10^{-5} \mathrm{M}$ solution in 49:49:2 (\% volume) water:methanol:acetic acid, focused through a region of differential pumping, and accumulated in a quadrupole ion trap (model C-125, R.M. Jordan, Grass Valley, CA) before being pulsed from the ion trap into the drift tube. The drift tube is $50.6 \mathrm{~cm}$ long with a uniform field of $12.8 \mathrm{~V} \cdot \mathrm{cm}^{-1}$ and is operated with $\sim 2.0$ torr of helium. Ions are gently extracted from the drift tube where they are focused into a quadrupole mass filter (ABB Extrel, Pittsburgh, PA) for selection of specific charge states. The mass-selected ions are accelerated to the desired kinetic energy and then injected into an octopole collision cell operated at a pressure of 


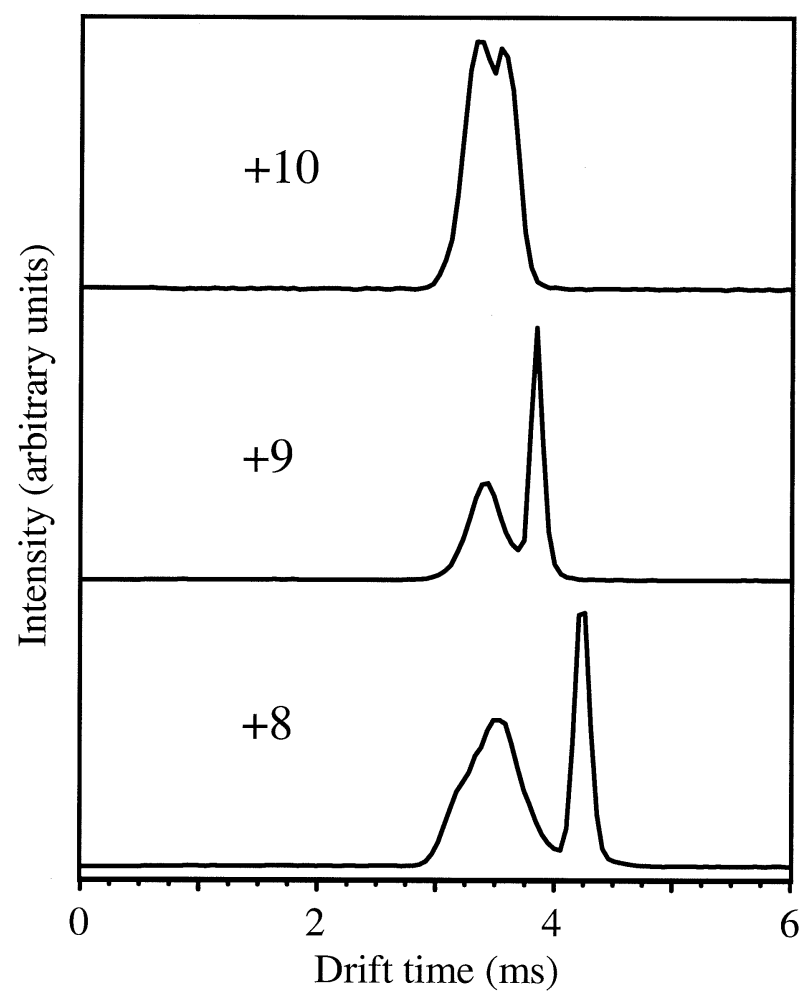

Figure 1. Drift time distributions for the +8 (bottom), +9 (middle), and +10 (top) charge states of ubiquitin.

0.17 mTorr of argon. Precursor and fragment ions that exit the collision cell are focused into the source of the orthogonal TOF mass spectrometer.

The ion mobility experiment operates on millisecond time scales, and the TOF experiment operates on microsecond time scales; therefore, it is possible to record multiple TOF spectra across each mobility-separated peak. We refer to this as a nested drift (flight) time experiment and have described the approach in detail elsewhere [43]. The resulting data sets acquired are presented as two-dimensional plots of the mass-tocharge $(m / z)$ ratios against drift time. For each experiment, the charge state of interest, each of the [M + $8 \mathrm{H}]^{8+}$ through $[\mathrm{M}+10 \mathrm{H}]^{10+}$ ions of ubiquitin, was mass selected with the quadrupole mass filter and subjected to collision-induced dissociation (CID) conditions in the octopole collision cell. Because dissociation occurs after the drift tube, fragment ions are coincident with their antecedent parent ions [42,44], allowing the mixtures of fragments to be assigned to precursor ions with identical mobilities. The +8 to +10 charge states of ubiquitin were chosen for these experiments because of the relatively well-resolved multiple conformers exhibited by these charge states, as well as the ability to efficiently fragment these ions.

\section{Results and Discussion}

Figure 1 shows drift time distributions for the +8 to +10 charge states of ubiquitin obtained upon $200 \mathrm{~V}$ injection of electrosprayed ions into the drift tube. Each distribution shows evidence for at least two types of conformations. Distributions for the +8 and +9 charge states each show a broad feature (associated with relatively high-mobility ions having more compact structures) and a narrower peak at longer times (associated with more open conformations). The +10 distribution also shows evidence for two peaks, although these are not fully resolved under the conditions of these experiments. These distributions are consistent with data that we reported previously for ubiquitin ions [39]. We also note that these data are identical (within the experimental reproducibility) before and after additions of the target gas for CID studies.

Figure 2 shows an example two-dimensional drift (flight) time data set for fragmentation of the $[\mathrm{M}+$ $8 \mathrm{H}]^{8+}$ ion. These data are obtained by injecting mobility-separated and $\mathrm{m} / \mathrm{z}$-selected ions into a collision cell using a $60 \mathrm{~V}$ potential. Mass spectra obtained by integration of regions of the data associated with the broad and narrow drift peaks are also shown. Examination of the fragmentation data for these different ion structures shows that the fragmentation spectra are very similar. That is, we observe the same number of fragment peaks for each conformer; additionally, both spectra exhibit fragment ion peaks at identical $\mathrm{m} / \mathrm{z}$ ratios as well as similar peak intensities.

A more detailed comparison of the fragmentation of these different conformers can be obtained by examining a plot that shows one spectrum divided by the other (the inset in Figure 2b). In this case, we have divided the integrated mass spectrum for the compact distribution of ions by that obtained for the elongated distribution. Over the $m / z$ range $\sim 1050$ to 1400 (the region where most fragment peaks are found), the ratio of the divided data is $0.9 \pm 0.3$ (one standard deviation), essentially a constant. The variations that are observed appear to be random fluctuations in the data. There is no evidence for enhancement of any fragmentation pathway from one of the precursor conformer types.

Additional data for other CID conditions and for other charge states of ubiquitin have been obtained. Figure 3 shows fragmentation mass spectra for different conformations of the +9 ions under two different CID conditions. These data also show many assignable peaks that vary in intensity depending upon the CID conditions used. However, in all cases, we find that for a given set of conditions, different conformations appear to form the same fragments and with the same intensities. We find that at collision cell potentials of 45 $\mathrm{V}$ and $56 \mathrm{~V}$ the ratio of the divided data is $1.1 \pm 0.4$ and $0.8 \pm 0.3$, respectively, over the $m / z$ ranges of 800 to 1200 and 800 to 1400 . The same is true of the different conformers formed for the +10 charge state (although we have not shown these data). Overall, we have studied this system over several months and during this time could find no evidence for different fragmentation efficiencies for different conformations of any of the ubiquitin charge states. 

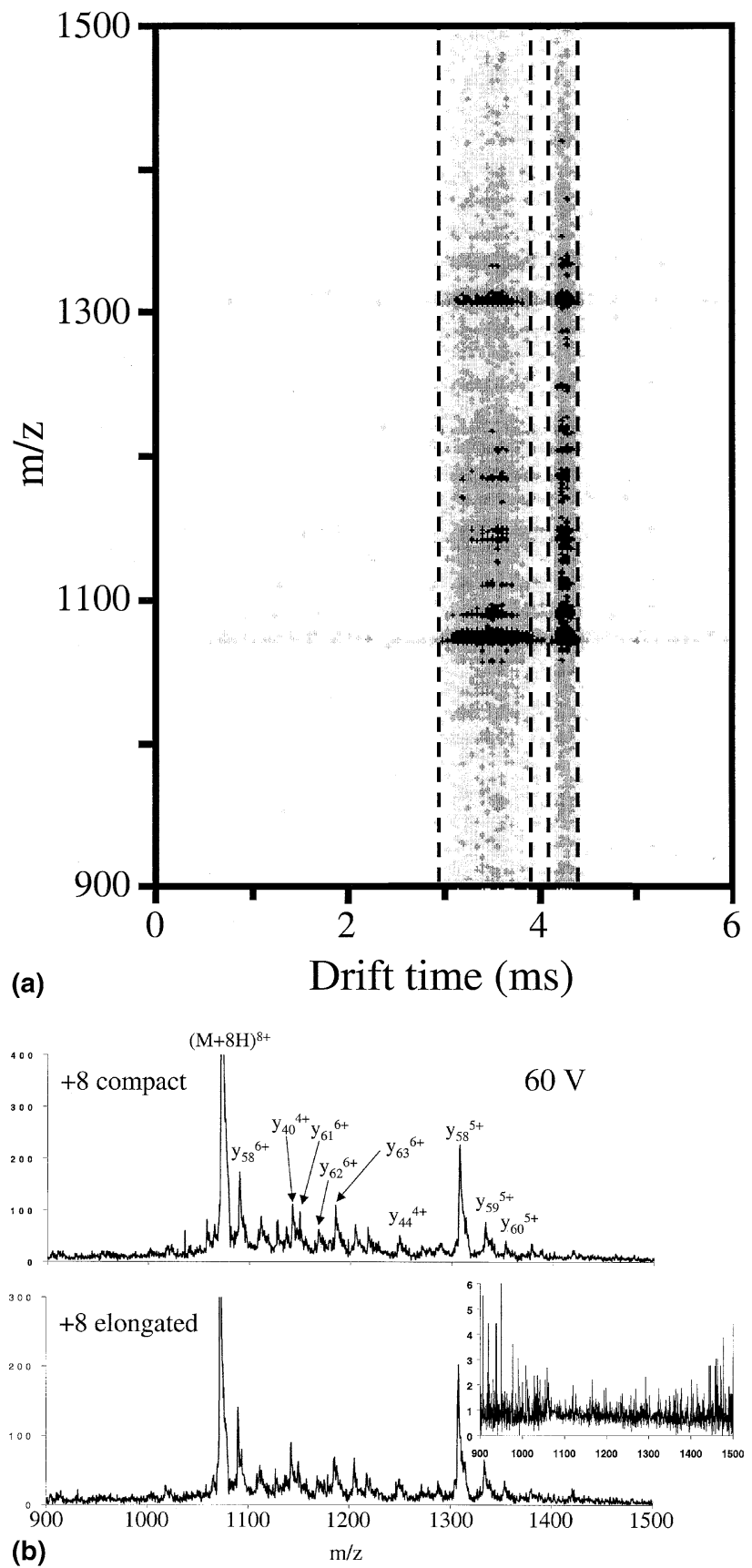

(b)

Figure 2. (a) A plot of a nested ion mobility/collision cell/timeof-flight spectrum for the mass selected +8 charge state of ubiquitin. Dissociation is promoted by injecting ions into the gas cell using a $60 \mathrm{~V}$ potential. The gas cell was filled with 0.17 mTorr of argon. The dashed lines are boundaries for the two resolved peaks shown in Figure 1. Integration across these regions allows fragment ion mass spectra for the resolved conformer types to be obtained. The intensities of peaks are indicated on a gray scale, with light gray being the least intense and black being the most intense features. (b) Fragmentation mass spectra of the compact (top) and elongated (bottom) conformers. The inset shows a ratio plot of compact and elongated conformer mass spectra obtained by dividing the two data sets.

A simple explanation for the lack of distinct fragment pathways within different conformations of a charge state of ubiquitin is that the initial activation step

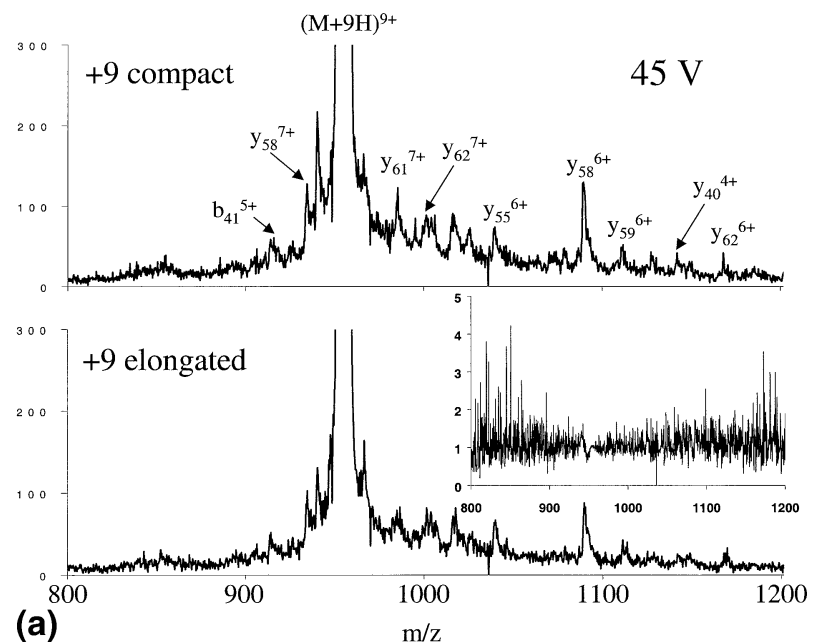

(a)
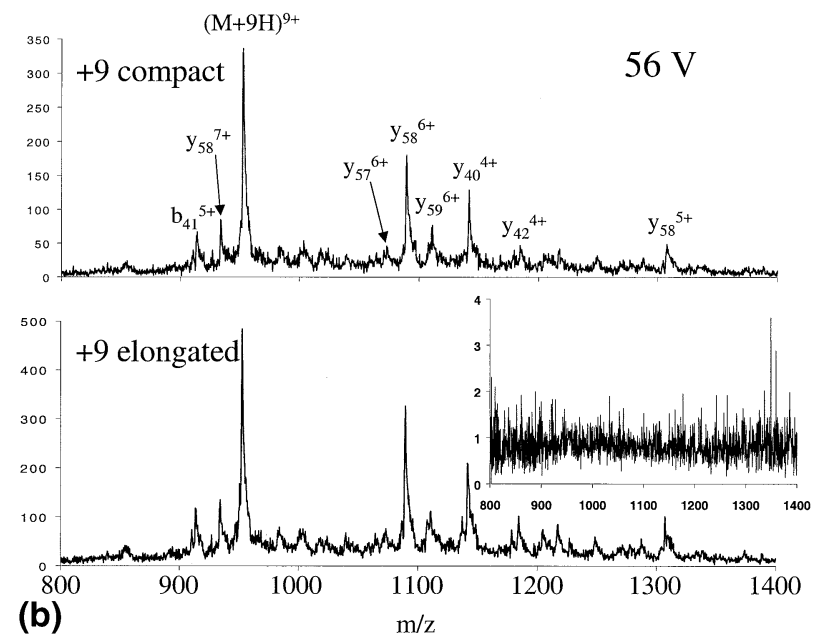

Figure 3. Fragmentation mass spectra of compact and elongated conformers of the +9 charge state of ubiquitin at collision voltages of (a) $45 \mathrm{~V}$, and (b) $56 \mathrm{~V}$ with parent and fragment ions labeled. The insets show a ratio plot obtained by dividing the fragmentation mass spectra for a compact and elongated ions.

converts different precursor conformations into similar structures. Previous ion mobility results have shown that compact conformations of protein ions can be favored when ions are gently injected into a drift tube; however, when the injection energy is increased, many of these ions rapidly unfold into more open structures $[45,46]$. This transition is the result of a rapid heating and cooling process that occurs at the front of the drift tube. As ions enter the high pressure region of the drift tube, their kinetic energies are thermalized by collisions with the buffer gas (ion kinetic energy is transferred into internal energy as a result of inelastic collisions with the buffer gas). Further collisions cool the ions to the temperature of the buffer gas. These experiments (and others $[24,47$ ) show that before enough energy is imparted to induce dissociation, structural transitions can occur. Similarly, different conformations introduced into the CID cell could convert into a similar structure prior to dissociation. In this case no differences in the fragmentation processes would be ex- 
pected. H/D exchange studies in an FT-ICR mass spectrometer have shown results that cytochrome $c$ ions partially unfolded prior to dissociation [11], supporting this hypothesis. We are currently examining a number of other systems, as well as different CID conditions in order to examine these issues in more detail.

\section{Summary and Conclusions}

An ion mobility/collisional activation/TOF mass spectrometry method has been used to examine fragmentation pathways for different conformers of ubiquitin ions. The results show that fragmentation patterns do not depend on the initial gas-phase ion conformation. The similarities in fragmentation patterns from different conformer types suggest that the different conformations convert to a common state prior to dissociation. This idea is supported by other work that shows that ubiquitin ions unfold to elongated structures when injected at high energies into a drift tube. If a similar transition occurs as the initial step during collisional activation in a gas cell, then differences in ion structure would not be apparent in the fragmentation spectra. The observation of similar results in other systems would have the practical consequence that conformation information from most folded proteins cannot be obtained from CID experiments.

Recently, several other types of studies suggest that information about parent ion conformation can be retained during the fragmentation process. Williams and coworkers have shown that the energetics of heme dissociation from myoglobin ions activated by blackbody infrared radiative dissociation (BIRD) reflect solution conditions prior to electrospray ionization [48]. Lebrilla and coworkers have used BIRD for dissociation of cyclodextrin:peptide complexes, and found that the peptide fragments more favorably than the complex dissociates [49]. Additionally, the technique of electron capture dissociation (ECD) [50-53] is currently thought to retain conformational information during the dissociation process. In the ECD process, the dissociation is believed to be very fast, such that internal energy is not randomized over the entire molecule (a nonergodic process), leaving noncovalent structure intact. Results have shown that laser heating of trapped ions affects the fragment ions observed with ECD, suggesting that changes in non-covalent structure (protein unfolding in the gas phase) and protein folding intermediates can be monitored using the technique [53].

\section{Acknowledgments}

This work is supported by a grant from the National Science Foundation (grant no. CHE-0078737). ERB is supported by a grant to the Indiana/Purdue University Indiana Instrumentation Institute (the III) through a grant from the Indiana 21st Century fund.

\section{References}

1. Winger, B. E.; Lightwahl, K. J.; Rockwood, A. L.; Smith, R. D. J. Am. Chem. Soc. 1992, 114, 5897-5898.

2. Suckau, D.; Shi, Y.; Beu, S. C.; Senko, M. W.; Quinn, J. P.; Wampler, F. M.; McLafferty, F. W. Proc. Natl. Acad. Sci. U.S.A. 1993, 90, 790-793.

3. Loo, R. R. O.; Smith, R. D. J. Am. Soc. Mass Spectrom. 1994, 5, 207-220.

4. Loo, R. R. O.; Winger, B. E.; Smith, R. D. J. Am. Soc. Mass Spectrom. 1994, 5, 1064-1071.

5. Williams, E. R. J. Mass Spectrom. 1996, 31, 831-842.

6. McLuckey, S. A.; Herron, W. J.; Stephenson, J. L.; Goeringer, D. E. J. Mass Spectrom. 1996, 31, 1093-1100.

7. Stephenson, J. L.; McLuckey, S. A. J. Am. Chem. Soc. 1996, 118, 7390-7397.

8. Stephenson, J. L.; McLuckey, S. A. J. Am. Chem. Soc. 1997, 119, 1688-1696.

9. Stephenson, J. L.; McLuckey, S. A. Anal. Chem. 1997, 69, 281-285.

10. Green, M. K.; Lebrilla, C. B. Mass Spectrom. Rev. 1997, 16, 53-71.

11. McLafferty, F. W.; Guan, Z. Q.; Haupts, U.; Wood, T. D.; Kelleher, N. L. J. Am. Chem. Soc. 1998, 120, 4732-4740.

12. Stephenson, J. L.; Schaaff, T. G.; McLuckey, S. A. J. Am. Soc. Mass Spectrom. 1999, 10, 552-556.

13. Freitas, M. A.; Hendrickson, C. L.; Emmett, M. R.; Marshall, A. G. Int. J. Mass Spectrom. 1999, 187, 565-575.

14. Schaaff, T. G.; Stephenson, J. L.; McLuckey, S. A. J. Am. Soc. Mass Spectrom. 2000, 11, 167-171.

15. Chowdhury, S. K.; Katta, V.; Chait, B. T. J. Am. Chem. Soc. 1990, 112, 9012-9013.

16. Loo, J. A.; Edmonds, C. G.; Udseth, H. R.; Smith, R. D. Anal. Chem. 1990, 62, 693-698.

17. Loo, J. A.; Loo, R. R. O.; Udseth, H. R.; Edmonds, C. G.; Smith, R. D. Rapid Commun. Mass Spectrom. 1991, 5, 101-105.

18. Ballard, K. D.; Gaskell, S. J. J. Am. Chem. Soc. 1992, 114, 64-71.

19. Wu, Q. Y.; Vanorden, S.; Cheng, X. H.; Bakhtiar, R.; Smith, R. D. Anal. Chem. 1995, 67, 2498-2509.

20. Dongre, A. R.; Jones, J. L.; Somogyi, A.; Wysocki, V. H. J. Am. Chem. Soc. 1996, 118, 8365-8374.

21. Vachet, R. W.; Asam, M. R.; Glish, G. L. J. Am. Chem. Soc. 1996, 118, 6252-6256.

22. Schnier, P. D.; Price, W. D.; Jockusch, R. A.; Williams, E. R. J. Am. Chem. Soc. 1996, 118, 7178-7189.

23. Tsaprailis, G.; Nair, H.; Somogyi, A.; Wysocki, V. H.; Zhong, W. Q.; Futrell, J. H.; Summerfield, S. G.; Gaskell, S. J. J. Am. Chem. Soc. 1999, 121, 5142-5154.

24. Li, J. W.; Taraszka, J. A.; Counterman, A. E.; Clemmer, D. E. Int. J. Mass Spectrom. 1999, 187, 37-47.

25. Schaaff, T. G.; Cargile, B. J.; Stephenson, J. L.; McLuckey, S. A. Anal. Chem. 2000, 72, 899-907.

26. Wells, J. M.; Stephenson, J. L.; McLuckey, S. A. Int. J. Mass Spectrom. 2000, 203, A1-A9.

27. Reid, G. E.; Wu, J.; Chrisman, P. A.; Wells, J. M.; McLuckey, S. A. Anal. Chem. 2001, 73, 3274-3281.

28. Loo, J. A.; He, J. X.; Cody, W. L. J. Am. Chem. Soc. 1998, 120, $4542-4543$.

29. Loo, J. A.; Quinn, J. P.; Ryu, S. I.; Henry, K. D.; Senko, M. W.; McLafferty, F. W. Proc. Natl. Acad. Sci. U.S.A. 1992, 89, 286289.

30. Loo, J. A.; Edmonds, C. G.; Smith, R. D. Anal. Chem. 1993, 65, $425-438$.

31. Beu, S. C.; Senko, M. W.; Quinn, J. P.; Wampler, F. M.; McLafferty, F. W. J. Am. Soc. Mass Spectrom. 1993, 4, 557-565.

32. Senko, M. W.; Speir, J. P.; McLafferty, F. W. Anal. Chem. 1994, $66,2801-2808$. 
33. Little, D. P.; Speir, J. P.; Senko, M. W.; O'Connor, P. B.; McLafferty, F. W. Anal. Chem. 1994, 66, 2809-2815.

34. Price, W. D.; Schnier, P. D.; Williams, E. R. Anal. Chem. 1996, 68, 859-866.

35. Jockusch, R. A.; Schnier, P. D.; Price, W. D.; Strittmatter, E. F.; Demirev, P. A.; Williams, E. R. Anal. Chem. 1997, 69, 11191126.

36. Stephenson, J. L.; McLuckey, S. A. Anal. Chem. 1998, 70, 3533-3544.

37. Zubarev, R. A.; Horn, D. M.; Fridriksson, E. K.; Kelleher, N. L.; Kruger, N. A.; Lewis, M. A.; Carpenter, B. K.; McLafferty, F. W. Anal. Chem. 2000, 72, 563-573.

38. Horn, D. M.; Zubarev, R. A.; McLafferty, F. W. Proc. Natl. Acad. Sci. U.S.A. 2000, 97, 10313-10317.

39. Valentine, S. J.; Counterman, A. E.; Clemmer, D. E. J. Am. Soc. Mass Spectrom. 1997, 8, 954-961.

40. Jarrold, M. F. In Cluster of Atoms and Molecules: Theory, Experiment, and Clusters of Atoms; Haberland, H, Ed.; SpringerVerlag: New York, 1994; pp 163-186.

41. Baer, T.; Mayer, P. M. J. Am. Soc. Mass Spectrom. 1997, 8, 103-115.

42. Hoaglund-Hyzer, C. S.; Li, J. W.; Clemmer, D. E. Anal. Chem. 2000, 72, 2737-2740.
43. Hoaglund, C. S.; Valentine, S. J.; Sporleder, C. R.; Reilly, J. P.; Clemmer, D. E. Anal. Chem. 1998, 70, 2236-2242.

44. Hoaglund-Hyzer, C. S.; Clemmer, D. E. Anal. Chem. 2001, 73, 177-184.

45. Valentine, S. J.; Clemmer, D. E. J. Am. Chem. Soc. 1997, 119, 3558-3566.

46. Shelimov, K. B.; Clemmer, D. E.; Hudgins, R. R.; Jarrold, M. F. J. Am. Chem. Soc. 1997, 119, 2240-2248.

47. Mao, Y.; Woenckhaus, J.; Kolafa, J.; Ratner, M. A.; Jarrold, M. F. J. Am. Chem. Soc. 1999, 121, 2712-2721.

48. Gross, D. S.; Zhao, Y. X.; Williams, E. R. J. Am. Soc. Mass Spectrom. 1997, 8, 519-524.

49. Penn, S. G.; He, F.; Lebrilla, C. B. J. Phys. Chem. B 1998, 102, 9119-9126.

50. Zubarev, R. A.; Kelleher, N. L.; McLafferty, F. W. J. Am. Chem. Soc. 1998, 120, 3265-3266.

51. Zubarev, R. A.; Kruger, N. A.; Fridriksson, E. K.; Lewis, M. A.; Horn, D. M.; Carpenter, B. K.; McLafferty, F. W. J. Am. Chem. Soc. 1999, 121, 2857-2862.

52. McLafferty, F. W.; Horn, D. M.; Breuker, K.; Ge, Y.; Lewis, M. A.; Cerda, B.; Zubarev, R. A.; Carpenter, B. K. J. Am. Soc. Mass Spectrom. 2001, 12, 245-249.

53. Horn, D. M.; Breuker, K.; Frank, A. J.; McLafferty, F. W. J. Am. Chem. Soc. 2001, 123, 9792-9799. 